\title{
Chiral packing of chiral quintuple layers polycatenated to give a three-dimensional network in the coordination polymer $\left[\mathrm{Co}_{5}(\mathrm{bpe})_{9}\left(\mathrm{H}_{2} \mathrm{O}\right)_{8}\left(\mathrm{SO}_{4}\right)_{4}\right]\left(\mathrm{SO}_{4}\right) \cdot 14 \mathrm{H}_{2} \mathrm{O}$ [bpe $=$ 1,2-bis(4-pyridyl)ethane]
}

\author{
Lucia Carlucci, $^{a}$ Gianfranco Ciani, $^{* b}$ Davide M. Proserpio ${ }^{b}$ and Silvia Rizzato ${ }^{b}$ \\ a Dipartimento di Biologia Strutturale e Funzionale, Università dell'Insubria, Via J. H. Dunant 3, 21100 Varese, \\ Italy \\ ${ }^{b}$ Dipartimento di Chimica Strutturale e Stereochimica Inorganica and Centro CNR, Via G. Venezian 21, 20133 \\ Milano, Italy.E-mail: davide@csmtbo.mi.cur.it
}

\author{
Received (in Basel, Switzerland) 13th March 2000, Accepted 26th May 2000 \\ Published on the Web 21st June 2000
}

The reaction of cobalt(II) sulfate with 1,2-bis(4-pyridyl)ethane gives a crystalline polymeric species comprised of chiral quintuple layers with five decks of linear chains rotated by $120^{\circ}$ from level to level, that are perpendicularly joined via five-metal helical motifs; furthermore, each of these two-dimensional frames is catenated by the neighbouring layers to give an overall three-dimensional chiral nanoporous architecture.

There is much current interest for the crystal engineering of coordination frameworks, ${ }^{1}$ not only because of their potential applications as zeolite-like materials, for molecular selection, ion exchange and catalysis, but also for their intriguing architectures, new topologies and intertwining phenomena. Some recent examples have shown that topological types unprecedented in inorganic chemistry and in minerals can be achieved within the area of networked coordination polymers. ${ }^{2}$ Moreover, new types of supramolecular intertwinings of infinite motifs have been discovered, that open interesting perspectives in the study of these materials. Particularly attractive are polymeric systems sustained by supramolecular entanglements reminiscent of the mechanical bonds in molecular rotaxanes and catenanes, exhibiting constituent motifs of dimensionality lower than that of the resulting overall architecture. Known examples include polyrotaxanes ${ }^{3}$ and polycatenated one-dimensional $^{4}$ and two-dimensional motifs. ${ }^{5}$ Species like these are expected to be of comparable robustness but more flexible than the usual networks entirely based on coordinative bonds with similar ligands. ${ }^{6}$ We report here on a new member of this class of polycatenated motifs, $\left[\mathrm{Co}_{5}(\mathrm{bpe})_{9}\left(\mathrm{H}_{2} \mathrm{O}\right)_{8}\left(\mathrm{SO}_{4}\right)_{4}\right]-$ $\left(\mathrm{SO}_{4}\right) \cdot 14 \mathrm{H}_{2} \mathrm{O}$ [bpe $=1$,2-bis(4-pyridyl)ethane] 1, that presents a new remarkable chiral three-dimensional architecture sustained by the catenation of chiral five-fold decked layers.

Compound $\mathbf{1}$ forms as beautiful flat hexagonal pink crystals by slow evaporation of solutions obtained on mixing bpe in ethanol and Co(II) sulfate in water, with metal-to-ligand molar ratios from $1: 1.7$ to $1: 2$. The crystals are stable in the air at room temperature for an extended time but start to lose solvated water molecules when heated above $c a .40{ }^{\circ} \mathrm{C}$. The powdered material obtained in good yields by fast evaporation of more concentrated solutions of the reagents corresponds to almost pure 1, as confirmed by X-ray powder diffraction methods

The crystal structure of $\mathbf{1} \dagger$ shows the presence of multiple layers containing five levels of one-dimensional linear polymers, all lying perpendicular to the crystallographic $c$ axis, that are formed by $\mathrm{Co}$ (II) ions joined by bpe ligands in a trans conformation (Co $\cdots$ Co distances of $13.69 \AA$ ). These polymers run on the five decks in different directions rotated by $120^{\circ}$ about the $c$ axis on passing from one level to the successive one, thus resulting in a $A B C A B$ sequence. The decks are connected along the normal direction via five-metal helical motifs, illustrated in Fig. 1, all exhibiting homochirality (left handed chirality for the crystal examined). The resulting chiral multilayer thus formed is schematically shown in Fig. 2.

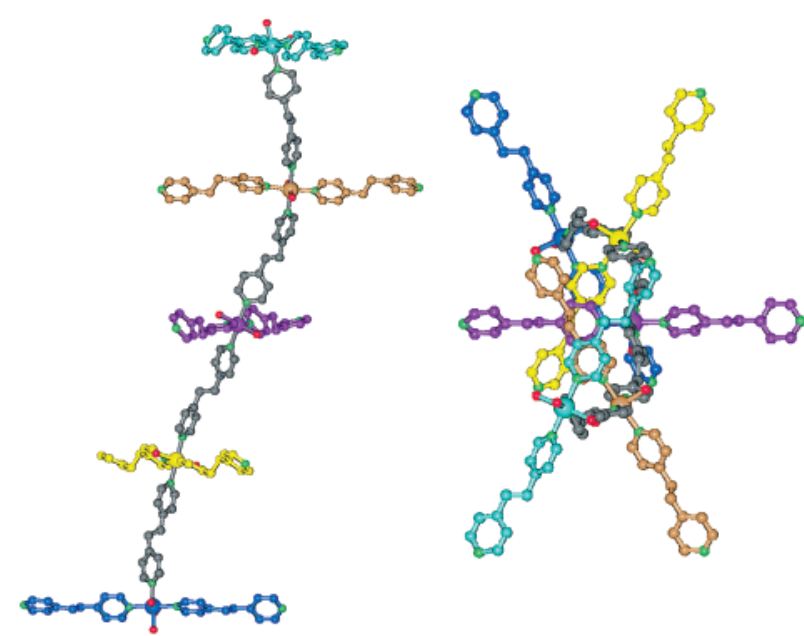

Fig. 1 A lateral (left) and a top (right) view of a single helical motif joining perpendicularly the quintuple layer (different colours are used to evidence the five decks). A two-fold axis passes through the central metal.

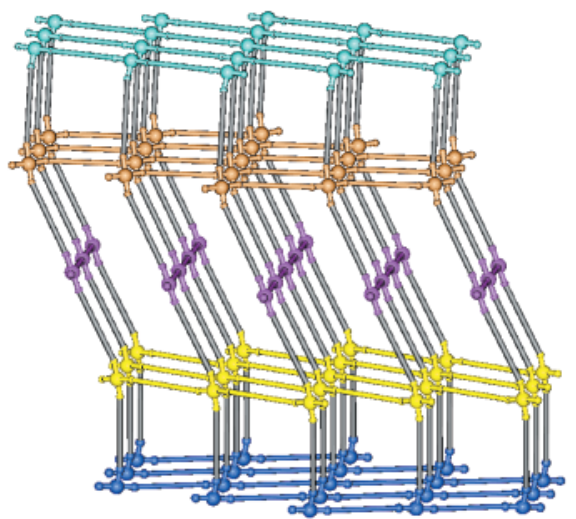

Fig. 2 A schematic view of a single five-decked layer with the same colours as in Fig. 1.

The cobalt ions of the two external levels are three-connected to bpe ligands (T-shaped geometry) while those of the three internal levels are four-connected to such ligands (square-planar geometry). The coordination spheres of the cobalt ions are completed by oxygen atoms of the sulfates and by water molecules (three mer $\mathrm{H}_{2} \mathrm{O}$ for the two external Co, two sulfates for the central cobalt, one $\mathrm{H}_{2} \mathrm{O}$ and one sulfate for the other two metal ions), resulting in all cases in distorted octahedral geometries. The five-metal helical chains are terminated at both ends by axial $\mathrm{H}_{2} \mathrm{O}$ ligands, i.e. the external 'surfaces' of the multilayers are covered by coordinated water molecules. Though other examples of multiple layers are known within coordination polymers, ${ }^{7}$ the two-dimensional motif of $\mathbf{1}$ is 
unique in terms of topology, number of decks and thickness (see Fig. 2). To our knowledge, it is the largest two-dimensional coordination polymer ever reported.

We can rationalize this two-dimensional polymeric motif as a five-layer section of the chiral three-dimensional fourconnected framework described by O'Keeffe as the 'dense' net. ${ }^{\text {Th }}$ This shows an $A B C A B C$ infinite sequence of levels and exhibits the unusual (759) topology. The first real example of this frame-work (three-fold interpenetrated) has been recently observed in a copper(II) polymeric species. ${ }^{2 a}$

The stacking distance between two adjacent levels of each quintuple layer lies in the range 12.26-13.10 $\AA$, so that the resulting overall height is as large as $50.5 \AA$. Identical layers are superimposed along the $c$ crystallographic axis, with an interlayer gap of $13.4 \AA$ (see Fig. 3). Two other sets of quintuple layers are generated by the $3_{1}$ symmetry axis, exhibiting sequences of their five levels of the $B C A B C$ and $C A B C A$ type, respectively, resulting in an overall chiral packing. The three sets are entangled in such a way that each layer is catenated not only by the two nearest neighbouring (upper and lower) layers but also by the two second nearest neighbouring ones, as illustrated in Fig. 3. Thus each layer is intertwined in a 'parallel fashion' $1 b$ with the other four, to give a fascinating polycatenated array. Infinite catenation of polymeric motifs represents a type of supramolecular entanglement that is still quite uncommon. Known examples include polycatenated onedimensional ladders that give two-dimensional ${ }^{4 b}$ or threedimensional arrays, ${ }^{4 a, c}$ as well as two-dimensional simple, ${ }^{5 c}$ double ${ }^{5 a}$ and quadruple layers, ${ }^{5 b}$ resulting in three-dimensional architectures. We have very recently reported also on a catenated three-dimensional system based on different polymeric motifs, i.e. one-dimensional ribbons of rings and twodimensional $(4,4)$-layers, that exhibits a peculiar sponge-like nanoporous behaviour. ${ }^{6} b$

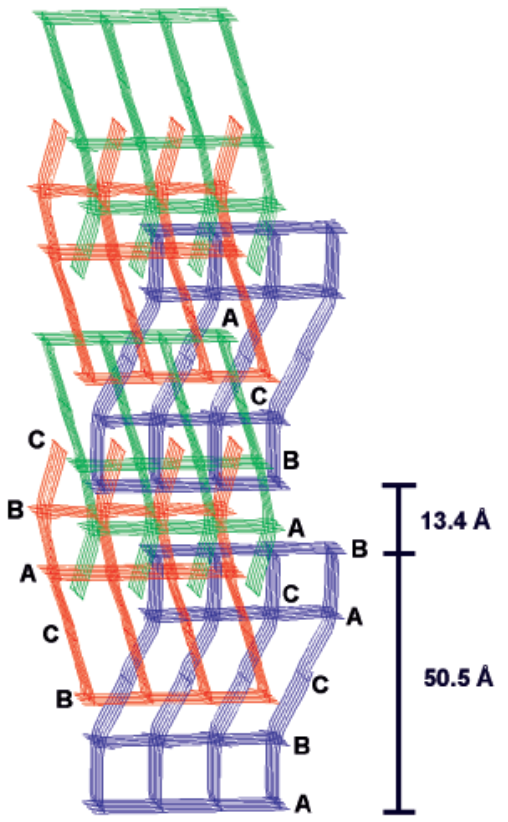

Fig. 3 The catenation of the two-dimensional quintuple layers. Three colours are used to evidence the different sets of layers generated by the $3_{1}$ axis. Inside one layer the two interpenetrating nearest neighbours are also entangled between themselves (the inner red and blue layers inside the central green one).

The polycatenation in compound $\mathbf{1}$ is not sufficient to fill all the free voids, corresponding to $c a$. the $19.6 \%$ of the cell volume (calculated by neglecting the chlatrated $\mathrm{H}_{2} \mathrm{O}$ molecules and the uncoordinated sulfates). These spaces are comprised of isolated cavities (three major cavities each of volume $c a .450 \AA^{3}$ ) containing solvated water molecules that can be removed by heating. Thermogravimetric analyses of $\mathbf{1}$, under a nitrogen flux, show that both the solvated and the coordinated water molecules are completely lost in a continuous fashion in the range $40-120{ }^{\circ} \mathrm{C}$. Above this point the product loses crystallinity and becomes unstable, $\$$ but if the samples are heated only up to $c a .80{ }^{\circ} \mathrm{C}$ the solvent loss ( $c a .8 \%$ of the total weight, corresponding approximately to the solvated water molecules) can be regained by exposure to water vapour overnight, as confirmed by TGA and XRPD.

Since catenation of polymeric frameworks can allow a certain flexibility of the whole architecture, it can potentially favour solvent exchange processes, as observed in previous examples. ${ }^{6}$ Compound 1 can be, therefore, considered as a model species for nanoporous arrays that present the additional features of non rigidity and chirality.

\section{Notes and references}

$\dagger$ Crystal data for compound 1: $\left[\mathrm{Co}_{5}(\mathrm{bpe})_{9}\left(\mathrm{H}_{2} \mathrm{O}\right)_{8}\left(\mathrm{SO}_{4}\right)_{4}\right]\left(\mathrm{SO}_{4}\right) \cdot 14 \mathrm{H}_{2} \mathrm{O}, M$ $=2829.43$, trigonal, $P 3_{1} 21$ (no. 152), $a=b=13.690$ (2), $c=63.880$ (9) $\AA, U=10368(3) \AA^{3}, Z=3, D_{\mathrm{c}}=1.359 \mathrm{Mg} \mathrm{m}^{-3}$, Mo-K $\alpha$ radiation $(\lambda=$ $0.71073 \AA), \mu(\mathrm{Mo}-\mathrm{K} \alpha)=0.745 \mathrm{~mm}^{-1}, 61444$ reflections measured, 9999 unique $\left(R_{\text {int }}=0.043\right)$, final $R 1=0.088$ for 9343 independent reflections [ $I$ $>2 \sigma(I)]$. The data collection was performed at $293 \mathrm{~K}$ on an SMART-CCD Bruker diffractometer, by the $\omega$-scan method, within the limits $2<\theta<24^{\circ}$. The structure was solved by direct methods (SIR97) and refined by fullmatrix least squares (SHELX97). The non coordinated sulfate anion is statistically distributed over two positions and was refined with half occupancy. One bpe ligand is statistically disordered by a two-fold axis passing through $\mathrm{N} 1$ and $\mathrm{N} 2$ and was refined using a half weight model. The handedness of the crystal was determined by testing the two enantiomeric models.

CCDC 182/1664. See http://www.rsc.org/suppdata/cc/b0/b002021o/ for crystallographic files in .cif format.

\$ Two other weight losses are observed. The first in the range $120-250{ }^{\circ} \mathrm{C}$ (ca. 19\%) corresponds approximately to three ligand molecules. The corresponding XRPD spectrum shows a completely different pattern arising from a new violet anhydrous phase. The second weight loss ( $c a$. 36-37\%), occurs in the range $280-430{ }^{\circ} \mathrm{C}$ and leads to a final residue of $\mathrm{CoSO}_{4}$, as evidenced by XRPD.

1 (a) B. F. Hoskins and R. Robson, J. Am. Chem. Soc., 1990, 112, 1546; (b) S. R. Batten and R. Robson, Angew. Chem., Int. Ed., 1998, 37, 1460; (c) O. M. Yaghi, H. Li, C. Davis, D. Richardson and T. L. Groy, Acc. Chem. Res., 1998, 31, 474; (d) M. Munakata, L. P. Wu and T. Kuroda-Sowa, Adv. Inorg. Chem., 1999, 46, 173; (e) P. J. Hagrman, D. Hagrman and J. Zubieta, Angew. Chem., Int. Ed., 1999, 38, 2638; (f) A. J. Blake, N. R. Champness, P. Hubberstey, W.-S. Li, M. A. Withersby and M. Schröder, Coord. Chem. Rev., 1999, 183, 117.

2 See e.g.: (a) L. Carlucci, G. Ciani, P. Macchi and D. M. Proserpio, Chem. Commun., 1998, 1837; (b) B. F. Abrahams, S. R. Batten, M. J. Grannas, H. Hamit, B. F. Hoskins and R. Robson, Angew. Chem., Int. Ed., 1999, 38, 1475 .

3 B. F. Hoskins, R. Robson and D. A. Slizys, J. Am. Chem. Soc., 1997, 119, 2952.

4 See, e.g.: (a) M. Fujita, Y. J. Kwon, O. Sasaki, K. Yamaguchi and K. Ogura, J. Am. Chem. Soc., 1995, 117, 7287; (b) A. J. Blake, N. R. Champness, A. Khlobystov, D. A. Lenenovkii, W.-S. Li and M. Schröder, Chem. Commun., 1997, 2027; (c) L. Carlucci, G. Ciani and D. M. Proserpio, J. Chem. Soc., Dalton Trans., 1999, 1799.

5 (a) F.-Q. Liu and T. D. Tilley, Inorg. Chem., 1997, 36, 5090; (b) L. Carlucci, G. Ciani, P. Macchi, D. M. Proserpio and S. Rizzato, Chem. Eur. J., 1999, 5, 237; (c) M.-L. Tong, X.-M. Chen, B.-H. Ye and L.-N. Ji, Angew. Chem., Int. Ed., 1999, 38, 2237.

6 (a) M. Fujita, O. Sasaki, K. Watanabe, K. Ogura and K. Yamaguchi, New J. Chem., 1998, 22, 189; (b) L. Carlucci, G. Ciani, D. M. Proserpio and S. Rizzato, Angew. Chem., Int. Ed., 2000, 40, 1506.

7 L. Carlucci, G. Ciani, D. M. Proserpio and A. Sironi, Angew. Chem., Int. Ed. Engl., 1995, 34, 1895; M. Kondo, T. Joshitomi, K. Seki, H. Matsuzaka and S. Kitagawa, Angew. Chem., Int. Ed. Engl., 1997, 36, 1725; K. N. Power, T. L. Hennigar and M. J. Zaworotko, New J. Chem., 1998, 22, 177.

8 M. O'Keeffe, Z. Kristallogr., 1991, 196, 21; M. O'Keeffe and B. G. Hyde, Crystal structures I: patterns and symmetry, Mineralogical Society of America, Washington, DC, 1996; see also M. O'Keeffe, Nature, 1998, 392, 879 . 